\title{
El "siempreverde" (Ligustrum lucidum), ¿Altera la composición de las comunidades de hongos micorrícicos arbusculares en el Chaco Serrano?
}

\author{
Valentina Borda ${ }^{\varpi}$; M. Noelia Cofré; Silvana Longo; Gabriel Grilli \& \\ Carlos Urcelay
}

Instituto Multidisciplinario de Biología Vegetal (CONICET, FCEFyN), Universidad Nacional de Córdoba, Córdoba, Argentina.

\begin{abstract}
RESUMEN. Las invasiones biológicas representan una gran amenaza para la biodiversidad y la integridad de los ecosistemas dado que alteran las comunidades bióticas. Entre las comunidades bióticas del suelo se destacan los hongos micorrícicos arbusculares (HMA), que establecen asociaciones simbióticas con la mayoría de las plantas terrestres. Las especies de HMA no responden del mismo modo a los cambios ambientales, por lo que se propuso la existencia de grupos de especies de HMA con estrategias ecológicas compartidas (ruderales, competidoras y estrés tolerantes). Las plantas invasoras son capaces de alterar las comunidades de HMA para su propio beneficio. Este podría ser el caso del "siempreverde" (Ligustrum lucidum), un árbol asiático que invade numerosos sectores de la Argentina y forma, en algunas zonas, bosques monoespecíficos densos. En este estudio se compararon las comunidades de esporas de HMA entre bosques monoespecíficos de siempreverde y bosques chaqueños serranos nativos. Se seleccionaron 6 áreas con ambos tipos de bosque. De cada área se tomaron muestras de suelo, a las que se les midieron características físico-químicas y se les extrajeron las esporas de HMA. De acuerdo con lo esperado, la composición de las comunidades de esporas de estos bosques monoespecíficos fue diferente a la presente en suelos de bosques nativos. La riqueza de morfoespecies de esporas de HMA no mostró diferencias entre ambos tipos de bosque, pero la abundancia total fue mayor en los suelos de bosque de siempreverde, que presentan un mayor número de esporas de especies ruderales. Este estudio provee evidencia que sugiere que la expansión del siempreverde altera las comunidades de esporas de HMA y algunas propiedades químicas del suelo. Estos cambios posiblemente sean parte del mecanismo que promueve la formación de bosques monoespecíficos.
\end{abstract}

[Palabras clave: planta invasora leñosa, grupos funcionales de hongos, propiedades del suelo, simbiosis]

\begin{abstract}
Aвstract. Does the "Glossy privet" (Ligustrum lucidum) alter the composition of the arbuscular mycorrhizal fungal communities in the Chaco Serrano? Biological invasions represent a major threat to biodiversity and the integrity of ecosystems, since they generate alterations in biotic communities. Among the biotic soil communities, arbuscular mycorrhizal fungi (AMF) are a major component establishing symbiotic associations with the majority of land plants. The HMA species do not respond in the same way to environmental changes; so, the existence of groups of HMA species with shared ecological strategies (ruderals, competitors and stresstolerants) was proposed. Invasive plants are capable of altering AMF communities for their own benefit. This could be the case of the "glossy privet" (Ligustrum lucidum), an Asian tree that invades important areas of Argentina and forms dense monospecific stands in certain locations. In this study, the HMA spore communities were compared between glossy privet monospecific forests and native Chaco Serrano forests. Six areas were selected with both types of forest. We obtained soil samples from which AMF spores were extracted and physicochemical properties were measured. As expected, the spore community composition differed between monospecific glossy privet stands and native forests. The HMA spore richness did not differ between both types of forest but the total abundance was higher in glossy privet stands that show a higher spore abundance of ruderal AMF species. This study provides evidence that suggests that L. lucidum expansion alters certain soil chemical properties and HMA spore communities. These changes may feedback on glossy privet growth and promote the formation of monospecific stands.
\end{abstract}

[Keywords: woody invasive plant, fungal functional groups, soil properties, symbiosis]

Editora asociada: Roxana Aragón
Recibido: 31 de Julio de 2019

Aceptado: 15 de Abril de 2020 


\section{INTRODUCCIÓN}

Las invasiones biológicas constituyen una de las amenazas más importantes para la biodiversidad y la integridad de los ecosistemas a nivel mundial (Sala et al. 2000). El establecimiento y la expansión de las poblaciones de plantas invasoras -en especial los árboles invasores (Richardson et al. 2014) - provoca alteraciones en las comunidades bióticas, tanto por encima como por debajo de la superficie del suelo, y sobre los procesos ecosistémicos. Estos impactos dependen de las características de la especie invasora y del ecosistema invadido, y pueden, a su vez, repercutir sobre los servicios ecosistémicos (Pyšek et al. 2012; Castro-Díez et al. 2019).

Entre las comunidades bióticas del suelo se destacan los hongos micorrícicos arbusculares (HMA), ya que establecen relaciones simbióticas con la mayoría de las plantas terrestres a nivel de sus raíces. Es probable que esta interacción constituya la relación simbiótica más extendida y ecológicamente importante del planeta (Fitter 2005; Smith and Read 2008). A cambio de carbono proveniente de la fotosíntesis, los simbiontes fúngicos facilitan el acceso a nutrientes a las plantas (sobre todo, fósforo) y proveen protección contra patógenos, entre otros beneficios. Como resultado de la interacción, los HMA suelen promover el crecimiento de las plantas hospedadoras (Smith and Read 2008).

A diferencia de lo que ocurre con otro tipo de simbiosis micorrícica como las ectomicorrizas (e.g., Moyano et al. 2020), tradicionalmente se acepta que la interacción HMA-planta es de tipo generalista. Por esta razón, se propuso que las especies exóticas pueden establecer asociaciones y beneficiarse de las comunidades de HMA nativas en los ecosistemas que invaden. En este sentido, la interacción con simbiontes generalistas y cosmopolitas constituye una estrategia mediante la cual las plantas pueden establecer poblaciones estables en regiones que se encuentran fuera de su área de distribución (Richardson et al. 2000; Núñez and Dickie 2014). No obstante, hay evidencia que muestra que las interacciones planta-HMA no se establecen al azar, sino que existe cierta selectividad entre los simbiontes (e.g., Eom et al. 2000; Helgason et al. 2002; Lovelock et al. 2003; Davison et al. 2011; Yang et al. 2012; Guisande-Collazo et al. 2016) y que el resultado de la simbiosis depende de la identidad de las especies vegetales y fúngicas, así como de las estrategias ecológicas de los simbiontes (Scheublin et al. 2007; Sikes et al. 2009; Chagnon et al. 2013; Yang et al. 2016; Urcelay et al. 2019).

Hay estudios que sugieren que las plantas son capaces de detectar, discriminar y recompensar con carbohidratos al simbionte fúngico más eficiente (Bever et al. 2009; Kiers et al. 2011). De este modo, el desarrollo de algunas especies de HMA "eficientes" puede promover el crecimiento y nutrición de especies de plantas que se convierten en dominantes de la comunidad vegetal (Reinhart and Callaway 2006). Esta retroalimentación positiva sería uno de los posibles mecanismos a través del cual las plantas invasoras se expanden en los ecosistemas que invaden (Zhang et al. 2010).

En estudios a campo se observó que las comunidades de HMA pueden ser resilientes frente a ciertos disturbios y resistentes a la remoción de algunos grupos funcionales de plantas (e.g., Urcelay et al. 2009). No obstante, cuando se comparan comunidades de plantas contrastantes en términos de diversidad, se suelen observar cambios en la diversidad de HMA (Burrows and Pfleger 2002). Además, se observó una relación positiva entre la riqueza de plantas y la de HMA (Hiiesalu et al. 2014). En la misma línea, los bosques monoespecíficos suelen albergar una menor diversidad y abundancia de esporas de HMA que los bosques mixtos; es decir, aquellos que cuentan con varias especies arbóreas (Sharmah and Jha 2014). Tal podría ser el efecto de ciertas especies invasoras que forman bosques monoespecíficos en las regiones que invaden. De hecho, se ha observado que las plantas invasoras pueden promover el desarrollo de algunos HMA más beneficiosos e incrementar la abundancia de esporas en el suelo (Greipsson and DiTommaso 2006).

Las especies de HMA no responden del mismo modo a los cambios ambientales. En este sentido, se propuso la existencia de grupos de especies de HMA con estrategias ecológicas compartidas (Chagnon et al. 2013). Por ejemplo, las especies de los clados Glomeraceae, Claroideoglomeraceae, Pacisporaceae, Diversisporaceae y Archaeosporaceae tendrían una estrategia ruderal (sensu Grime 1977), caracterizada por alta tasa de crecimiento, alta colonización intra-radical, producción temprana de esporas y baja densidad de hifas en el suelo (Maherali and Klironomos 2007). Existe evidencia que muestra que estas especies 
ruderales suelen ser generalistas en relación a los hospedadores y resistentes o capaces de recuperarse rápidamente frente a cambios en las comunidades vegetales y modificaciones en parámetros químicos del suelo (van der Heyde et al. 2017). Más aun, en ecosistemas del centro y sur de Argentina se ha observado que los clados Glomeraceae, Claroideoglomeraceae y Pacisporaceae no son afectadas por los disturbios tales como el fuego, el pastoreo y la fragmentación de bosques (Longo et al. 2016; Dudinszky et al. 2019). En cambio, a las especies pertenecientes a Gigasporaceae se les asigna una estrategia competidora, caracterizada por producir micelio extraradical extenso con mayor capacidad de exploración del suelo y solubilización de P (Chagnon et al. 2013). A su vez, las especies de Acaulosporaceae son consideradas tolerantes al estrés con bajas tasas de crecimiento, micelio durable, resistencia a la acidez y a las bajas temperaturas. Debido a estos caracteres, estos últimos dos grupos funcionales son poco tolerantes a los disturbios y a cambios en la composición de especies vegetales (Longo et al. 2016; van der Heyde et al. 2017; Dudinszky et al. 2019).

Las comunidades vegetales de las montañas del centro de la Argentina están constituidas principalmente por bosques mixtos correspondientes al Distrito Chaqueño Serrano. La gran mayoría de las especies vegetales de estos bosques forman micorrizas arbusculares (Fracchia et al. 2009; Tecco et al. 2013). Sectores relevantes de estas montañas fueron ocupados por el siempreverde (Ligustrum lucidum), una especie arbórea de origen asiático invasora en el centro y norte argentino. Como la mayoría de las especies leñosas nativas, esta leñosa invasora establece relaciones simbióticas con hongos micorrícicos arbusculares (Tecco et al. 2013). En los ecosistemas montañosos del centro de la Argentina, su área de distribución aumentó drásticamente en los últimos años (GavierPizarro et al. 2012), y en varios sectores forma bosques monoespecíficos densos (Hoyos et al. 2010). Hay estudios que muestran que los bosques de siempreverde tienen el potencial de afectar la composición de hongos saprófitos (Fernández et al. 2017) y las propiedades químicas y la descomposición de la broza (Aragón et al. 2014a y b; Furey et al. 2014). Sin embargo, se desconoce el impacto que pueden tener estos bosques sobre las comunidades bióticas del suelo, en particular los HMA.
En este estudio se examinó y comparó la estructura de las comunidades de esporas de HMA (diversidad morfotaxonómica y funcional entre bosques monoespecíficos de siempreverde y bosques nativos). Debido a que los procesos de invasión por plantas pueden afectar las propiedades físico-químicas del suelo (Ehrenfeld 2010) y que éstas pueden influir en la composición de HMA, se midieron las propiedades químicas de los suelos y se determinó la clase textural.

En base a lo expuesto, es razonable postular que la presencia de plantas exóticas invasoras que forman bosques monoespecíficos promoverá cambios en las comunidades bióticas del suelo y particularmente una disminución en la riqueza de HMA. Se predice que a) las comunidades de esporas de HMA en bosques monoespecíficos de siempreverde serán menos diversas que las de bosques nativos; b) las esporas pertenecientes a familias con estrategia ruderal serán más abundantes mientras que las pertenecientes a familias con estrategia competidora y estrés tolerante serán menos abundantes en los bosques monoespecíficos de siempreverde.

\section{Materiales y Métodos}

\section{Área de estudio}

El estudio se llevó a cabo en las serranías de la provincia de Córdoba correspondientes al distrito Serrano de la provincia fitogeográfica del Chaco (Cabrera 1976). Esta región se encuentra entre 500 y 1500 m s. n. m. (Lutti et al. 1979), las precipitaciones presentan una media anual de $750 \mathrm{~mm}$ y están concentradas en la temporada cálida (octubre a abril). Las temperaturas medias máximas y mínimas anuales oscilan entre los $26^{\circ} \mathrm{C}$ y los $10{ }^{\circ} \mathrm{C}$, respectivamente (Moglia and Giménez 1998).

Se seleccionaron sitios que presentaron áreas muy próximas de bosques monoespecíficos de siempreverde y bosques nativos (Figura 1). Los nativos se caracterizan por la presencia de numerosas leñosas nativas, tales como la dominante Lithraea molleoides (Anacardiaceae) y otras como Zanthoxylum coco (Rutaceae), Celtis spp. (Celtidaceae), Schinus fasciculatus (Anacardiaceae), Vachellias sp. (Fabaceae), Condalia spp. (Rhamnaceae), Ruprechtia apetala (Polygonaceae), entre otras (Cabrera 1976; Cabido and Zack 1999; Zeballos et al. 2014). La fisonomía corresponde a la de un 


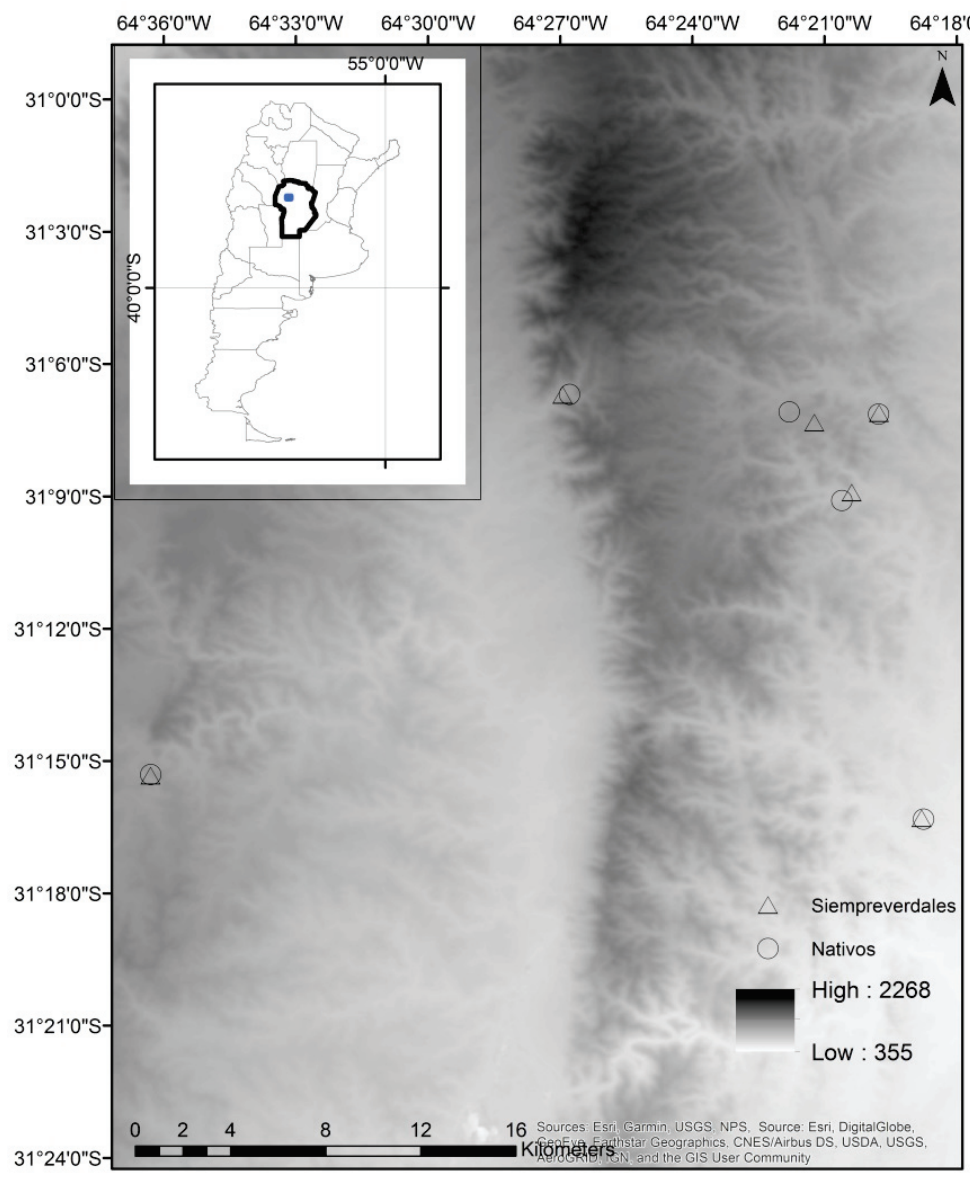

Figura 1. Ubicación geográfica del área de estudio y los bosques muestreados. La escala de grises indica la elevación en m s. n. m.

Figure 1. Geographical location of study area and forests sampled. The gray scale indicates the elevation in m a.s. 1 . bosque abierto a semicerrado, con estratos arbustivo y herbáceo (Estrabou 1983; Cabido and Zak 1999).

\section{Diseño experimental}

Se seleccionaron seis sitios; en cada uno de ellos se ubicaron dos bosques contiguos: nativo y monoespecífico de siempreverde. Los bosques de siempreverde (siempreverdales) se encuentran presentes en las Sierras de Córdoba desde su introducción en 1900 (Río and Achával 1904) y el área de distribución de éstos creció cincuenta veces en el período que va desde 1983 a 2006 (Gavier-Pizarro et al. 2012).

Entre el 9 y 16 de septiembre de 2017 se colectaron cinco muestras de suelo en cada una de las doce áreas de bosque (seis de siempreverdales y seis de nativos). Cada muestra se obtuvo de los $10 \mathrm{~cm}$ superficiales del suelo (removiendo previamente la hojarasca). En el caso de los bosques nativos, la muestra se tomó a $30 \mathrm{~cm}$ de la base de molle (L. molleoides), la especie arbórea más abundante del bosque serrano nativo, a los fines de estandarizar el muestreo y ajustar las comparaciones entre bosques a un muestreo similar bajo una misma especie; de igual modo se realizó en los siempreverdales, pero en la base de siempreverde. Para ambas especies se seleccionaron árboles de individuos adultos con un diámetro aproximado de al menos $20 \mathrm{~cm}$ y separados entre sí por una distancia mínima de aproximadamente 3-4 $\mathrm{m}$. Las cinco muestras tomadas en cada bosque fueron mezcladas para los análisis posteriores.

\section{Propiedades físico-químicas de los suelos}

En las muestras de suelo también se realizaron las siguientes determinaciones físico-químicas: materia orgánica (\%), nitrógeno total (\%), relación carbono/nitrógeno $(\mathrm{C}: \mathrm{N})$, nitrato (ppm), amoníaco (ppm), fósforo (ppm), pH, conductividad eléctrica y clase textural de los suelos de los dos tipos de bosque estudiados (Sparks 1996). Los análisis se realizaron en el Laboratorio edafológico de la Facultad de Agronomía (Universidad Nacional de Córdoba, Argentina). 
Caracterización de las comunidades de HMA

Para comparar las comunidades de HMA entre los bosques monoespecíficos de siempreverde y bosques nativos, de la mezcla de las cinco muestras tomadas en cada bosque se separaron dos porciones, una de $50 \mathrm{~g}$ de peso fresco y otra de $30 \mathrm{~g}$ de peso fresco. La porción de $30 \mathrm{~g}$ se secó en estufa hasta alcanzar peso seco constante, a fin de obtener la diferencia entre peso seco y peso fresco, ya que los datos obtenidos se expresan en valores de peso seco (cantidad de esporas por $100 \mathrm{~g}$ de peso seco). La porción de $50 \mathrm{~g}$ se utilizó para extraer y luego identificar la comunidad de esporas de HMA de cada una de las muestras de suelo siguiendo el protocolo propuesto por Brundrett et al. (1996). Las esporas fueron separadas y montadas en alcohol polivinílico (PVA) y PVA + Melzer, para su posterior identificación a nivel de especie -o en su defecto a nivel de género- y cuantificación mediante el uso de un microscopio óptico. La identificación se realizó en base a caracteres morfológicos microscópicos tales como tamaño, color, tipo de ornamentaciones de la pared, tipo de inserción de la hifa subtendida, forma de la misma, cantidad de paredes y láminas de estas. Para ello, se consultó información suministrada en la página del "InternationalCultureCollection of Arbuscular and Vesicular-Arbuscular Mycorrhizal Fungi" (http://invam.caf.wvu.edu) y materiales depositados en el Herbario CORD. Se usaron las clasificaciones propuestas por Redecker et al. (2013) y Schüßler and Walker (2010) y los nombres se asignaron de acuerdo a la lista de especies de HMA que se actualizó en septiembre de 2018 en http:/ /www.amfphylogeny.com. Se consideraron sólo aquellas esporas visualmente viables (turgentes y sin daño aparente).

\section{Análisis de datos}

Propiedades químicas de los suelos. Se utilizaron modelos lineales generales para determinar si existen diferencias en las variables químicas del suelo según el factor fijo "tipo de bosque" con dos niveles (siempreverdal y nativo).

Caracterización de las comunidades de HMA. Se construyeron curvas de rarefacción y de acumulación de especies para evaluar la profundidad del muestreo (Figura 6). Para analizar la variación de la comunidad de esporas de HMA en los distintos tipos de bosque se construyeron matrices de distancia basadas en disimilitud de BrayCurtis (proporciones) y Sorensen (presenciaausencia) utilizando la función vegdist (las especies raras fueron excluidas). Se analizó la variación en la composición de comunidades de esporas entre ambos tipos de bosque mediante un análisis de la varianza multivariado con permutaciones (PERMANOVA). Se controló la dispersión multivariada (i.e., análogo a la homogeneidad de varianza) de los grupos mediante la función betadisper (Anderson 2006). Se llevó a cabo un escalamiento multidimensional no métrico (NMDS) para realizar un ordenamiento de los bosques según su composición de morfoespecies de esporas. A su vez se realizaron regresiones de las variables químicas del suelo con los dos ejes del NMDS. Todos los análisis se realizaron con el paquete vegan (Oksanen et al. 2019). Además, se realizó un análisis de especies indicadoras (Dufrene and Legendre 1997) para cada tipo de bosque con el paquete labdsv (Roberts 2016).

Se utilizaron modelos lineales generales para analizar las diferencias entre la riqueza y la abundancia total según el factor fijo "tipo de bosque" (dos niveles). A su vez, utilizando los mismos modelos, se analizaron diferencias entre las abundancias de los tres grupos funcionales de morfoespecies encontradas en cada uno de los sitios para el factor "tipo de bosque". Para establecer dichos grupos funcionales las familias de HMA fueron consideradas según la información provista en http://www.amf-phylogeny.com. El tamaño del bosque fue incluido en los modelos como covariable y luego se eligieron aquellos modelos estadísticos que presentaran un índice de AIC más bajo para analizar las variables mencionadas. Los análisis estadísticos se llevaron a cabo en R Coreteam (2019).

\section{Resultados}

\section{Variables físico-químicas de los suelos}

De los parámetros químicos medidos, el amonio y el nitrato (marginalmente) fueron más altos en bosques nativos que en siempreverdales, mientras que el pH mostró valores marginalmente significativos más altos en siempreverdales (Tabla 1). En relación a las propiedades físicas, los suelos de los doce bosques muestreados correspondieron a la clase textural franco-arenosa. 
Tabla 1. Variables químicas de los suelos de los dos tipos de bosque estudiados. Media \pm error estándar (E.E.); $n=6$. Table 1. Chemical soil variables from both types of forest studied. Mean \pm standard error (E.E.); $n=6$.

\begin{tabular}{lccccccccc}
\hline & \multicolumn{7}{c}{ Siempreverdal } & \multicolumn{3}{c}{ Nativo } \\
& Media & \pm & E.E & Media & & E.E & $F$ & $P$ \\
\hline Materia orgánica (\%) & 15.07 & \pm & 1.59 & 13.53 & \pm & 1.15 & 0.61 & 0.452 \\
Nitrógeno total (\%) & 0.73 & \pm & 0.07 & 0.67 & \pm & 0.06 & 0.44 & 0.523 \\
Relación C:N & 11.88 & \pm & 0.28 & 11.68 & \pm & 0.15 & 0.4 & 0.5397 \\
Nitrato (ppm) & 103.98 & \pm & 59.88 & 258 & \pm & 40.01 & 4.57 & 0.058 \\
Amonio (ppm) & 22.83 & \pm & 7.87 & 51 & \pm & 7.91 & 6.37 & 0.03 \\
Fósforo (ppm) & 24.9 & \pm & 5.31 & 35.58 & \pm & 5.73 & 1.87 & 0.201 \\
pH & 7.28 & \pm & 0.14 & 6.87 & \pm & 0.13 & 4.82 & 0.053 \\
Conductividad eléctrica (dS/m) & 1.03 & \pm & 0.08 & 1.21 & \pm & 0.11 & 4.82 & 0.053 \\
\hline
\end{tabular}

Caracterización de las comunidades de HMA

Se identificaron un total de seis mil quinientas sesenta esporas correspondientes a cincuenta y nueve morfoespecies de HMA. De estas, cincuenta y tres se asignaron a especies conocidas mientras que seis fueron identificadas a nivel genérico. Nueve morfoespecies raras (es decir aquellas que ocurrieron sólo una vez) fueron excluidas de los análisis de riqueza y composición.

La composición de las comunidades de esporas, considerando la abundancia relativa de las morfoespecies, fue diferente entre tipos de bosque (pseudo- $F=2.99 ; P=0.03$ ). Por el contrario, no se observaron diferencias al considerar presencia-ausencia ( $p s e u d o-F=1.19$; $P=0.31$ ). El NMDS muestra claramente dos grupos (Figura 2). La conductividad eléctrica fue la única variable edáfica que mostró una relación significativa con la composición de las comunidades fúngicas (Figura 2). La

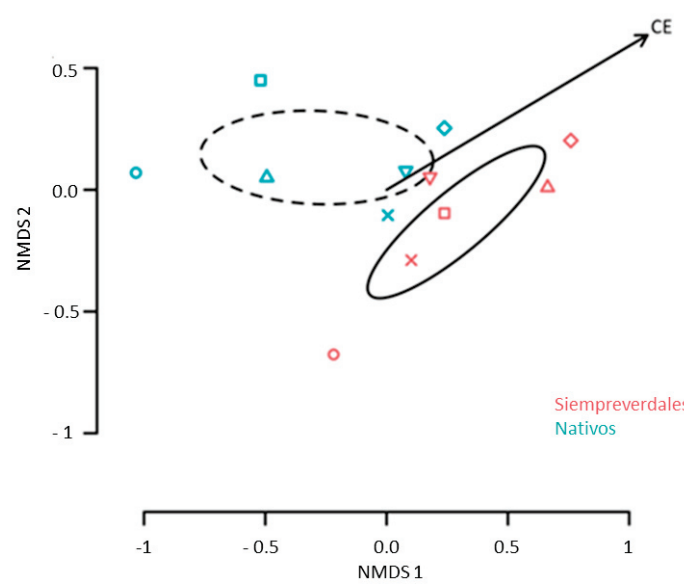

Figura 2. NMDS de los bosques según la composición de morfoespecies de esporas de HMA. Las formas iguales indican cercanía geográfica. CE: Conductividad eléctrica. Estrés $=0.07 ; n=6$.

Figure 2. NMDS of the forests according to the composition of the AMF spores. Equal forms indicate geographic closeness. CE: Electrical conductivity. Stress=0.07; $n=6$. dispersión multivariada de la varianza (i.e., para proporciones y presencia-ausencia) no presentó diferencias entre los grupos de los distintos tipos de bosque.

Dos especies fueron indicadoras de los siempreverdales: Septoglomus constrictum (valor indicador $=0.9 ; P=0.003$ ) y Glomus tortuosum (valor indicador $=0.73 ; P=0.041$ ) y una del bosque nativo: Pacispora sp. 1 (valor indicador $=0.92 ; P=0.02$ ). Los modelos estadísticos que no incluyeron al tamaño de bosque como covariable fueron seleccionados por presentar un menor índice de AIC. La riqueza de morfoespecies no mostró diferencias entre ambos tipos de bosque $(F=0.18 ; P=0.6771$ ) (Figura 3$)$, mientras que la abundancia total fue significativamente mayor en bosques de siempreverde $(F=5.32$; $P=0.0438$ ) (Figura 4).

Las esporas de HMA correspondientes a especies con estrategia ruderal (pertenecientes a Glomeraceae, Claroideoglomeraceae, Pacisporaceae, Diversisporaceae y Archaeosporaceae) fueron las más abundantes

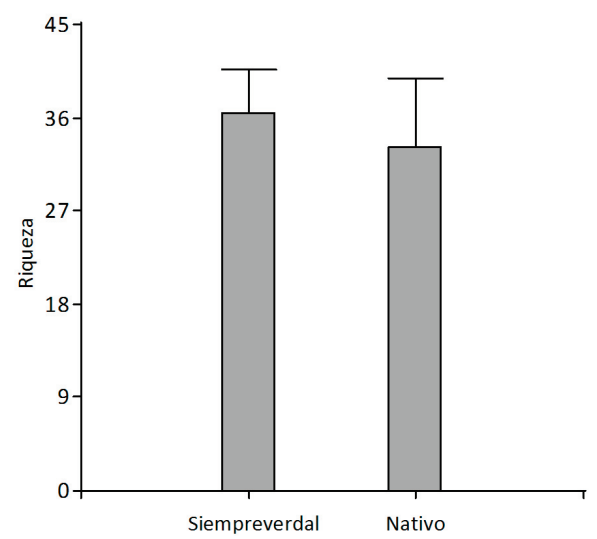

Figura 3. Riqueza de esporas de HMA en ambos tipos de bosque. Valores medios y error estándar; $n=6$.

Figure 3. Richness of HMA spores in both types of forests. Mean values and standard error; $n=6$. 


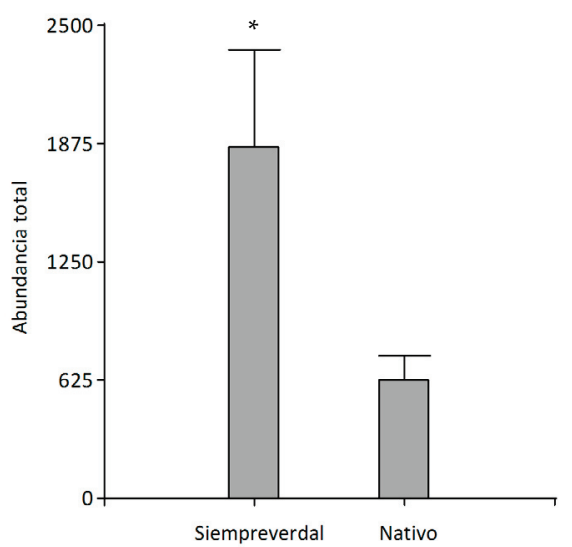

Figura 4. Abundancia total de esporas de HMA en $100 \mathrm{~g}$ de peso seco de suelo en ambos tipos de bosque. Valores medios y error estándar; $n=6$; $: P<0.05$.

Figure 4. Total abundance of HMA spores in $100 \mathrm{~g}$ dry weight of soil in both types of forests. Mean values and standard error; $n=6$; *: $P<0.05$.

en ambos tipos de bosque. Dicho grupo funcional estuvo constituido principalmente por Glomeraceae, tanto en siempreverdales como en bosques nativos. No obstante, la abundancia de esporas de este grupo funcional fue mayor en los suelos de bosques invadidos $(F=5.51 ; P=0.0408)$ (Figura 5). Las esporas correspondientes a los otros dos grupos funcionales mostraron una abundancia muy baja y no se encontraron diferencias entre ambos tipos de bosque $(F=0.08$ y $P=0.7844$ para los estrés tolerantes, $F=1.00$ y $P=0.3409$ para los competidores).

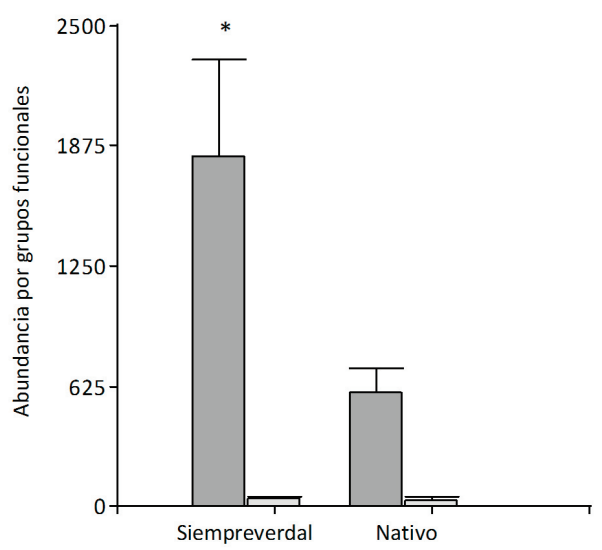

Figura 5. Abundancias de morfoespecies de esporas de HMA ruderales: en gris oscuro y estrés tolerantes: en gris claro en ambos tipos de bosque, la abundancia de esporas de especies competidoras no se observa debido a que los valores son muy bajos. Valores medios y error estándar; $n=6$; *: $P<0.05$.

Figure 5. Abundances of ruderal: in dark gray, and tolerant stress: in light gray HMA morphospecies in both types of forest, the abundance competing species spores is not observed because the values are very low. Mean values and standard error; $n=6$; * $P<0.05$.
Glomus aureum fue la especie más abundante en ambos tipos de bosque (Tabla 2 y 3). En los siempreverdales fue seguida por $S$. constrictum, la cual no se encontró dentro de las más abundantes en los bosques nativos mixtos. Acaulospora denticulata y G. trufemii fueron exclusivas de bosques nativos, mientras que Archeaospora trappei, Funneliformis mosseae, G. brohultii, G. warcupii y $S$. deserticola fueron exclusivas de los siempreverdales.

\section{DisCUSIÓN}

Se identificaron cincuenta morfoespecies de HMA a partir del estudio de las esporas presentes en el suelo (sin contar las especies raras), cuarenta y cinco en bosques nativos y cuarenta y ocho en siempreverdales. Estos valores son mayores que los treinta y dos observados por Longo et al. (2014) en el bosque nativo. Asimismo, los valores obtenidos aquí son similares a los registrados recientemente en pastizales, áreas cultivadas y bosques de espinal nativo en la región pampeana, obtenidos tanto a partir de la identificación morfológica de esporas (Cofré et al. 2017) como a partir de técnicas de secuenciación de DNA de esporas e hifas extra-radicales (Faggioli et al. 2019). Esto sugiere que la metodología utilizada aquí ha sido sensible para la detección de la mayoría de las morfoespecies de HMA presentes en bosques nativos y siempreverdales en la región de estudio.

Las comunidades de HMA presentes en siempreverdales que se encuentran en los ecosistemas montañosos del centro de la Argentina no habían sido estudiadas con anterioridad. De acuerdo con la hipótesis planteada, la composición de las comunidades de esporas de HMA de estos bosques monoespecíficos es diferente a la presente en suelos de bosques nativos, aunque contrariamente a lo predicho, no se observó una menor riqueza en los bosques invadidos. Sin embargo, la abundancia de esporas fue más alta en siempreverdales por presentar un mayor desarrollo de especies ruderales en estos bosques monoespecíficos. Estos resultados coinciden con uno de los pocos estudios donde se examinan las comunidades de esporas de HMA en bosques invadidos por leñosas (Greipsson and DiTommaso 2006). Es importante señalar que si bien los bosques nativos presentan numerosas especies leñosas, arbustivas y herbáceas (Giorgis et al. 2017), el suelo recolectado en estos bosques fue obtenido debajo de la copa de los molles a $30 \mathrm{~cm}$ del tronco a fines de estandarizar 
Tabla 2. Abundancias relativas de especies de esporas en ambos tipos de bosque. Media \pm error estándar (E.E.); $n=6$. Las familias de HMA fueron consideradas según la información provista por http:/ / www.amfphylogeny.com.

Table 2. Relative abundances of spore species in both types of forests. Mean \pm standard error (E.E.); $n=6$. The AMF families were considered according to the information provided by http:/ / www.amfphylogeny.com.

\begin{tabular}{|c|c|c|c|c|c|c|c|}
\hline \multicolumn{8}{|l|}{ HMA } \\
\hline \multirow[b]{2}{*}{ Familia } & \multirow[b]{2}{*}{ Especie } & \multicolumn{3}{|c|}{ Siempreverdal } & \multicolumn{3}{|c|}{ Nativo } \\
\hline & & Media & & E.E. & Media & & E.E. \\
\hline \multirow[t]{11}{*}{ Acaulosporaceae } & Acaulospora capsicula & 0.01 & \pm & 0.01 & 0.01 & \pm & 0.01 \\
\hline & A. delicata & 7.60E-04 & \pm & $4.90 \mathrm{E}-04$ & 0.01 & \pm & $3.80 \mathrm{E}-03$ \\
\hline & A. denticulata & 0 & \pm & 0 & $8.00 \mathrm{E}-04$ & \pm & 8.00E-04 \\
\hline & A. excavata & $6.30 \mathrm{E}-04$ & \pm & $6.30 \mathrm{E}-04$ & $3.60 \mathrm{E}-03$ & \pm & $3.10 \mathrm{E}-03$ \\
\hline & A. lacunosa & $8.70 \mathrm{E}-04$ & \pm & $5.50 \mathrm{E}-04$ & 0.01 & \pm & 0.01 \\
\hline & A. laevis & 3.00E-03 & \pm & $1.50 \mathrm{E}-03$ & 4.00E-04 & \pm & 4.00E-04 \\
\hline & A. rehmii & 6.90E-04 & \pm & 3.30E-04 & $3.20 \mathrm{E}-03$ & \pm & $3.20 \mathrm{E}-03$ \\
\hline & A. scrobiculata & $1.50 \mathrm{E}-03$ & \pm & $1.20 \mathrm{E}-03$ & $1.60 \mathrm{E}-03$ & \pm & $1.20 \mathrm{E}-03$ \\
\hline & A. spinosa & $1.50 \mathrm{E}-03$ & \pm & $1.50 \mathrm{E}-03$ & 0.01 & \pm & $4.00 \mathrm{E}-03$ \\
\hline & A. tuberculata & 7.60E-04 & \pm & 4.90E-04 & $1.40 \mathrm{E}-03$ & \pm & $1.40 \mathrm{E}-03$ \\
\hline & A. undulata & $6.30 \mathrm{E}-04$ & \pm & 6.30E-04 & 2.60E-03 & \pm & $1.60 \mathrm{E}-03$ \\
\hline Archaeosporaceae & Archaeospora trappei & 4.00E-04 & \pm & 3.10E-04 & 0 & \pm & 0 \\
\hline \multirow[t]{5}{*}{ Diversisporaceae } & Diversispora aurantia & 3.20E-03 & \pm & 2.10E-03 & 0.02 & \pm & 0.02 \\
\hline & D. eburnea & 0 & \pm & 0 & 6.90E-04 & \pm & $6.90 \mathrm{E}-04$ \\
\hline & D. pustulata & $4.00 \mathrm{E}-03$ & \pm & $1.20 \mathrm{E}-03$ & $2.60 \mathrm{E}-03$ & \pm & $1.40 \mathrm{E}-03$ \\
\hline & D. spurca & $1.10 \mathrm{E}-03$ & \pm & $1.10 \mathrm{E}-03$ & $3.00 \mathrm{E}-03$ & \pm & $3.00 \mathrm{E}-03$ \\
\hline & Redeckera fulva & $9.00 \mathrm{E}-05$ & \pm & $9.00 \mathrm{E}-05$ & 0 & \pm & 0 \\
\hline \multirow[t]{4}{*}{ Claroideoglomeracea } & Claroideoglomus claroideum & 3.90E-03 & \pm & $1.20 \mathrm{E}-03$ & 0.01 & \pm & $2.30 \mathrm{E}-03$ \\
\hline & C. etunicatum & 0.01 & \pm & $1.30 \mathrm{E}-03$ & 0.01 & \pm & $3.80 \mathrm{E}-03$ \\
\hline & C. lamellosum & 0.01 & \pm & $1.80 \mathrm{E}-03$ & 0.01 & \pm & $4.70 \mathrm{E}-03$ \\
\hline & C. luteum & 0.05 & \pm & 0.02 & 0.05 & \pm & 0.01 \\
\hline \multirow[t]{9}{*}{ Glomeraceae } & Funneliformis geosporus & 0.01 & \pm & 0.01 & 0.01 & \pm & 0.01 \\
\hline & F. mosseae & $6.50 \mathrm{E}-04$ & \pm & 3.20E-04 & 0 & \pm & 0 \\
\hline & Glomus aggregatum & 0.11 & \pm & 0.03 & 0.09 & \pm & 0.03 \\
\hline & G. ambisporum & 0.01 & \pm & $1.90 \mathrm{E}-03$ & 3.90E-03 & \pm & $3.90 \mathrm{E}-03$ \\
\hline & G. aureum & 0.22 & \pm & 0.07 & 0.4 & \pm & 0.06 \\
\hline & G. badium & 0.02 & \pm & 0.01 & 0.03 & \pm & 0.01 \\
\hline & G. brohultii & $1.70 \mathrm{E}-03$ & \pm & $1.20 \mathrm{E}-03$ & 0 & \pm & 0 \\
\hline & G. clavisporum & 3.60E-04 & \pm & 2.30E-04 & 0.01 & \pm & 0.01 \\
\hline & G. corimbiforme & 0.02 & \pm & 0.01 & 0.01 & \pm & $3.70 \mathrm{E}-03$ \\
\hline
\end{tabular}
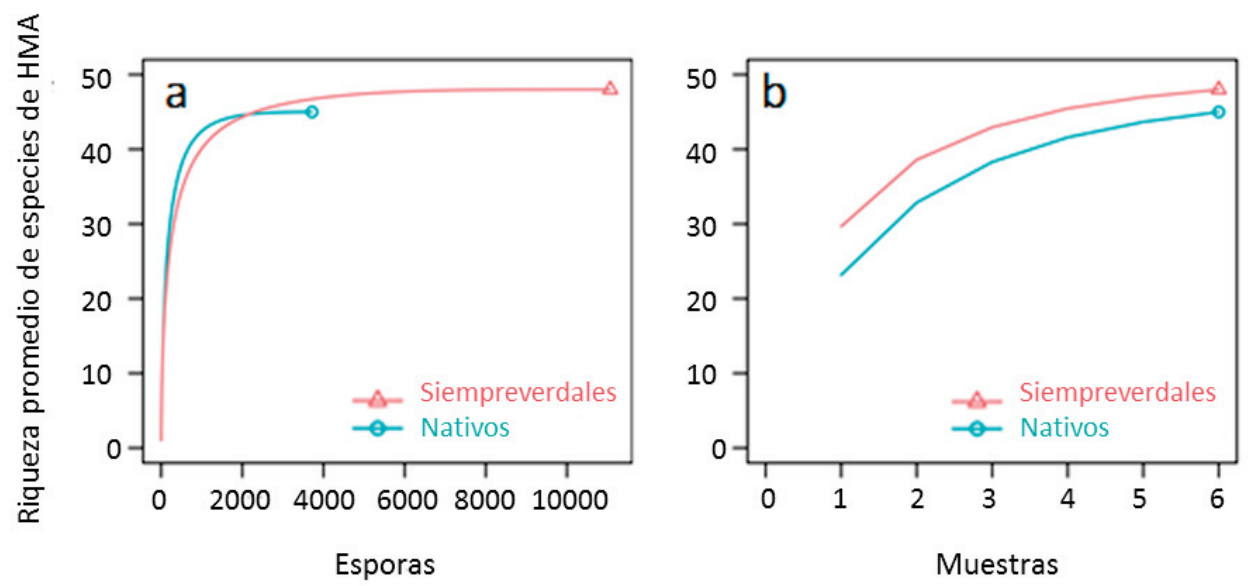

Figura 6. Curvas de a) rarefacción y de b) acumulación de morfoespecies de HMA en sitios de bosque nativo y siempreverdal.

Figure 6. a) rarefaction and b) accumulation curves of HMA morphospecies in native and evergreen forest sites. 
Tabla 3. Abundancias relativas de especies de esporas en ambos tipos de bosque. Media \pm error estándar (E.E.); $n=6$. Las familias de HMA fueron consideradas según la información provista por http://www.amfphylogeny.com/ amphylo_taxonomy.html.

Table 3. Relative abundances of spore species in both types of forest. Mean \pm standard error (E.E); $n=6$. The AMF families were considered according to the information provided by http://www.amfphylogeny.com.

\begin{tabular}{|c|c|c|c|c|c|c|c|}
\hline \multirow{2}{*}{$\begin{array}{l}\text { HMA } \\
\text { Familia }\end{array}$} & \multirow[b]{2}{*}{ Especie } & \multicolumn{3}{|c|}{ Siempreverdal } & \multicolumn{3}{|c|}{ Nativo } \\
\hline & & Media & & E.E. & Media & & E.E. \\
\hline \multirow[t]{25}{*}{ Glomeraceae } & G. diaphanum & $1.60 \mathrm{E}-03$ & \pm & 8.90E-04 & 2.10E-03 & \pm & $2.10 \mathrm{E}-03$ \\
\hline & G. glomerulatum & 0 & \pm & 0 & 7.80E-04 & \pm & 7.80E-04 \\
\hline & G. invermauim & 4.40E-03 & \pm & 2.90E-03 & 0.01 & \pm & 0.01 \\
\hline & G. macrocarpum & 0.02 & \pm & 0.01 & 0.01 & \pm & 0.01 \\
\hline & G. melanosporum & 0.07 & \pm & 0.03 & 0.07 & \pm & 0.02 \\
\hline & G. microagregatum & 0.01 & \pm & $2.80 \mathrm{E}-03$ & $2.80 \mathrm{E}-03$ & \pm & $2.40 \mathrm{E}-03$ \\
\hline & G. microcarpum & 0.05 & \pm & 0.02 & 0.08 & \pm & 0.05 \\
\hline & G. pustulatum & $1.70 \mathrm{E}-03$ & \pm & $6.00 \mathrm{E}-04$ & $1.50 \mathrm{E}-03$ & \pm & $1.10 \mathrm{E}-03$ \\
\hline & G. rubiforme & $3.10 \mathrm{E}-03$ & \pm & 3.10E-03 & 0 & \pm & 0 \\
\hline & G. segmentatum & $9.00 \mathrm{E}-05$ & \pm & $9.00 \mathrm{E}-05$ & 0 & \pm & 0 \\
\hline & G. spinuliferum & $3.10 \mathrm{E}-04$ & \pm & $3.10 \mathrm{E}-04$ & $2.10 \mathrm{E}-03$ & \pm & $1.60 \mathrm{E}-03$ \\
\hline & G. tortuosum & 0.02 & \pm & $4.50 \mathrm{E}-03$ & 0.01 & \pm & $4.50 \mathrm{E}-03$ \\
\hline & G. trufemii & 0 & \pm & 0 & $1.30 \mathrm{E}-03$ & \pm & $8.60 \mathrm{E}-04$ \\
\hline & G. warcupii & $1.20 \mathrm{E}-03$ & \pm & $9.10 \mathrm{E}-04$ & 0 & \pm & 0 \\
\hline & Glomus sp. 1 & 2.00E-04 & \pm & $2.00 \mathrm{E}-04$ & 0 & \pm & 0 \\
\hline & Glomus sp. 2 & 3.10E-04 & \pm & 3.10E-04 & $9.90 \mathrm{E}-04$ & \pm & $6.50 \mathrm{E}-04$ \\
\hline & Glomus sp. 3 & $1.50 \mathrm{E}-03$ & \pm & $1.50 \mathrm{E}-03$ & 0 & \pm & 0 \\
\hline & Rhizophagus clarus & $2.00 \mathrm{E}-03$ & \pm & $1.70 \mathrm{E}-03$ & $1.80 \mathrm{E}-03$ & \pm & $8.60 \mathrm{E}-04$ \\
\hline & R. fasciculatus & $3.80 \mathrm{E}-03$ & \pm & $2.70 \mathrm{E}-03$ & $2.50 \mathrm{E}-03$ & \pm & $1.20 \mathrm{E}-03$ \\
\hline & R. intraradices & 0.05 & \pm & 0.02 & 0.02 & \pm & 0.01 \\
\hline & Sclerocistis sp. 1 & 0.01 & \pm & $2.90 \mathrm{E}-03$ & $9.50 \mathrm{E}-04$ & \pm & $6.10 \mathrm{E}-04$ \\
\hline & Septoglomus constrictum & 0.23 & \pm & 0.06 & 0.03 & \pm & 0.01 \\
\hline & S. deserticola & $1.10 \mathrm{E}-03$ & \pm & $9.20 \mathrm{E}-04$ & 0 & \pm & 0 \\
\hline & S. viscosum & 0.01 & \pm & $3.80 \mathrm{E}-03$ & 0.01 & \pm & $3.50 \mathrm{E}-03$ \\
\hline & Simiglomus hoi & 0.02 & \pm & 0.01 & 0.01 & \pm & 0.01 \\
\hline Gigasporaceae & Gigaspora sp. 1 & $2.00 \mathrm{E}-04$ & \pm & 2.00E-04 & 0 & \pm & 0 \\
\hline \multirow[t]{2}{*}{ Pacisporaceae } & Pacispora chimbonobambusae & $1.60 \mathrm{E}-04$ & \pm & $1.60 \mathrm{E}-04$ & 0 & \pm & 0 \\
\hline & Pacispora sp. 1 & 3.60E-03 & \pm & $2.10 \mathrm{E}-03$ & 0.04 & \pm & 0.02 \\
\hline incertae sedis & Entrophospora infrequens & 0.01 & \pm & $1.50 \mathrm{E}-03$ & 0.01 & \pm & 3.70E-03 \\
\hline
\end{tabular}

el muestreo. Por lo tanto, las comunidades caracterizadas se encuentran influidas por esta especie arbórea y posiblemente se haya subestimado la riqueza de HMA en bosque nativo.

Septoglomus constrictum (Glomeraceae) fue una de las especies más abundantes en los siempreverdales e indicadora de los suelos de estos bosques. Longo et al. (2014) observaron que la abundancia de esporas de dicha especie es mayor en bosques que previamente sufrieron incendios. En conjunto, estos resultados sugieren que la especie es favorecida por disturbios (e.g., incendios) y cambios sustanciales en las comunidades vegetales (e.g., los que provocan las invasiones de plantas exóticas).

De acuerdo con lo esperado, la abundancia de esporas de especies ruderales fue mayor en los siempreverdales. Sin embargo, no hubo diferencias en la abundancia de las competidoras y de las estrés tolerantes. En el caso de estas dos estrategias, hay que interpretar con precaución los datos ya que los valores de abundancia fueron muy bajos. La baja abundancia de Acualosporaceae y particularmente de Gigasporaceae encontrada en ambos tipos de bosque concuerdan con lo observado en otros estudios en la misma región (Urcelay et al. 2009; Longo et al. 2014; Dudinszky et al. 2019). En este sentido, análisis moleculares complementarios de los suelos permitirían contrastar los resultados de este estudio y confirmar estos patrones.

Las diferencias en las comunidades de HMA entre ambos tipos de bosque podrían estar relacionadas al éxito del siempreverde para invadir y perpetuarse en nuevas regiones. Es posible que esta especie invasora obtenga mayores beneficios de las comunidades de HMA favorecidas por sus bosques 
monoespecíficos, a modo de retroalimentación positiva (Zhang et al. 2010).

Está bien documentado que los procesos de invasión por plantas afectan propiedades químicas del suelo y el ciclado de nutrientes (e.g., Hobbie 1992; Liao et al. 2008; Ehrenfeld 2010). Análisis globales indican que las invasiones por plantas tienden a afectar de manera positiva la disponibilidad de amonio y nitrato en el suelo (Liao et al. 2008), aunque esto varía entre ecosistemas y formas de vida de las plantas (Hobbie 1992; Ehrenfeld 2010). La mayor disponibilidad de nitrógeno - casi el doble de amonio y nitrato- en los suelos del bosque nativo puede deberse a que las tasas de descomposición de la broza son significativamente más altas que en bosques monoespecíficos de siempreverde (Furey at al. 2014). Por otro lado, también es posible que el menor contenido de nitrógeno en los siempreverdales se deba a una mayor tasa de incorporación de este nutriente por la especie invasora (Aragón et al. 2014b). La calidad de la broza, dada por la relación carbono/nitrógeno $(\mathrm{C}: \mathrm{N})$, también podría estar afectando la disponibilidad de estos nutrientes en el suelo (Hobbie 1992). En este sentido, la presencia de numerosas leñosas caducifolias sumada a la presencia de un denso estrato herbáceo en bosques nativos con valores de C:N más bajos que el siempreverde, cuyos bosques monoespecíficos carecen de estratos herbáceo y arbustivo, también explicarían la mayor disponibilidad de nitrógeno en el suelo de bosque nativo.

Los cambios en las comunidades de HMA también pueden atribuirse a las diferencias en las propiedades químicas del suelo (EgertonWarburton and Allen 2000; Fitzsimons et al. 2008; Jansa et al. 2014). En línea con otros estudios (Egerton-Warburton and Allen 2000; Treseder 2004; Grilli et al. 2012), la abundancia de esporas fue más alta donde la disponibilidad de $\mathrm{N}$ fue más baja. Estos resultados sugieren que las diferencias promovidas por el siempreverde en la abundancia de esporas de HMA podrían, en parte, estar mediadas por los efectos de este árbol invasor en las variables químicas del suelo.

Asimismo, la composición de comunidades de esporas se relacionó significativamente con la conductividad eléctrica. Si bien hubo diferencias marginalmente significativas de esta variable entre tipos de bosques, la magnitud de esa diferencia promedio (i.e., 0.8 $\mathrm{dS} / \mathrm{m}$ ) no parece ser significativa en términos biológicos, al menos si la comparamos con importantes gradientes naturales de salinidad (e.g., Aliasgharzadeh et al. 2001). Por lo tanto, no podemos realizar mayores inferencias sobre dicha relación.

\section{CONCLUSIONES}

Este trabajo que replica bosques nativos y siempreverdales a escala de paisaje provee evidencia que sugiere que la expansión del siempreverde y la consecuente formación de bosques monoespecíficos alteran las comunidades de esporas de HMA y algunas propiedades químicas del suelo. Estos cambios posiblemente afecten la composición de plantas en dichos bosques y eventualmente sean parte del mecanismo que promueve la formación de bosques monoespecíficos.

Agradecimientos. Agradecemos al Consejo Nacional de Investigaciones Científicas y Técnicas (CONICET) y a la Universidad Nacional de Córdoba (U.N.C.) los cuales han proporcionado el equipamiento y espacio de trabajo utilizados en este estudio. Este programa de investigación es financiado por la Secretaría de Ciencia y Tecnología (SECyT), la Universidad Nacional de Córdoba y el Ministerio de Ciencia y Tecnología de Córdoba. Se agradece también la colaboración de Nicolás Marro en la extracción de las esporas. Finalmente agradecemos los constructivos comentarios de un revisor anónimo y de la editora R. Aragón que mejoraron el aporte de este trabajo.

\section{REFERENCIAS}

Aliasgharzadeh, N., S. N. Rastin, H. Towfighi, and A. Alizadeh. 2001. Occurrence of arbuscular mycorrhizal fungi in saline soils of the Tabriz Plain of Iran in relation to some physical and chemical properties of soil. Mycorrhiza 11: 119-122. https://doi.org/10.1007/s005720100113.

Anderson, M. J. 2006. Distance-based tests for homogeneity of multivariate dispersions Biometrics 62:245 53. https: / /doi.org/10.1111/j.1541-0420.2005.00440.x.

Aragón, R., L. Montti, M. Ayup, and R. Fernández. 2014a. Exotic species as modifiers of ecosystem processes: Litter decomposition in native and invaded secondary forests of NW Argentina. Acta Oecologica 54:21-28. https:/ /doi.org/ 10.1016/j.actao.2013.03.007.

Aragón, R., J. Sardans, and J. Peñuelas. 2014b. Soil enzymes associated with carbon and nitrogen cycling in invaded and native secondary forests of northwestern Argentina. Plant and Soil 384:169-183. https:/ /doi.org/10.1007/s11104014-2192-8. 
Bever, J. D., S. C. Richardson, B. M. Lawrence, J. Holmes, and M. Watson. 2009. Preferential allocation to beneficial symbiont with spatial structure maintains mycorrhizal mutualism. Ecology Letters 12:13-21. https:/ / doi.org/10.1111/ j.1461-0248.2008.01254.x.

Brundrett, M., N. Bougher, B. Dell, and T. Grove. 1996. Working With Mycorrhizas in Forestry and Agriculture. ACIAR Monograph 32. Canberra, Australia: Australian Centre for International Agricultural Research.

Burrows, R. L., and F. L. Pfleger. 2002. Arbuscular mycorrhizal fungi respond to increasing plant diversity. Canadian Journal of Botany 80:120-130. https://doi.org/10.1139/b01-138.

CastronDíez, P., A. S. Vaz, J. S. Silva, M. Van Loo, Á. Alonso, C. Aponte, A. Bayón, P. J. Bellingham, M. C. Chiuffo, N. DiManno, K. Julian, S. Kandert, N. La Porta, H. Marchante, H. G. Maule, M. Margaret, M. Mayfield, D. Metcalfe, M. C. Monteverdi, M. A. Núñez, R. Ostertag, I. M. Parker, D. A. Peltzer, L. J. Potgieter, M. Raymundo, D. Rayome, O. ReismanロBerman, D. M. Richardson, R. E. Roos A. Saldaña, R. T. Shackleton, A. Torres, M. Trudgen, J. Urban, J. R. Vicente, M. Vilà, T. Ylioja, R. D. Zenni, and O. Godoy. 2019. Global effects of nonØnative tree species on multiple ecosystem services. Biological Reviews 94:1477-1501. https:/ / doi.org/10.1111/brv.12511.

Cabido, M. R., and M. R. Zak. 1999. Vegetación del Norte de Córdoba. Secretaria de Agricultura, Ganadería y Recursos Renovables de Córdoba.

Cabrera, A. L. 1976. Regiones fitogeográficas argentinas. Enciclopedia Argentina de Agricultura y Jardinería. Tomo II, Fasc. I, Buenos Aires.

Chagnon, P. L., R. L. Bradley, H. Maherali, and J. N. Klironomos. 2013. A trait-based framework to understand life history of mycorrhizal fungi. Trends in Plant Science 18:484-491. https://doi.org/10.1016/j.tplants.2013.05.001.

Cofré, M. N., A. E. Ferrari, A. Becerra, L. Domínguez, L. G. Wall, and C. Urcelay. 2017. Effects of cropping systems under noltill agriculture on arbuscular mycorrhizal fungi in Argentinean Pampas. Soil Use and Management 33: 364-378. https://doi.org/10.1111/sum.12349.

Davison, J., M. Opik, J. T. Daniell, M. Moora, and M. Zoobel. 2011. Arbuscular mycorrhizal fungal communities in plant roots are not random assemblages. FEMS Microbiology Ecology 78:103-115. https://doi.org/10.1111/j.15746941.2011.01103.x.

Dudinszky, N., M. N. Cabello, A. A. Grimoldi, S. Schalamuk, and R. A. Golluscio. 2019. Role of Grazing Intensity on Shaping Arbuscular Mycorrhizal Fungi Communities in Patagonian Semiarid Steppes. Rangeland Ecology and Management 72:692-699. https://doi.org/10.1016/j.rama.2019.02.007.

Dufrene, M., and P. Legendre. 1997. Species assemblages and indicator species: the need for a flexible asymmetrical approach. Ecological Monographs 67:345-366. https://doi.org/10.2307/2963459. https://doi.org/10.1890/00129615(1997)067[0345:SAAIST]2.0.CO;2.

Egerton-Warburton, L. M., and E. B. Allen. 2000. Shifts in arbuscular mycorrhizal communities along an anthropogenic nitrogen deposition gradient. Ecological Applications 10:484-496. https:/ / doi.org/10.1890/1051-0761(2000)010[0484: SIAMCA]2.0.CO;2.

Ehrenfeld, J. G. 2010. Ecosystem consequences of biological invasions. Annual Review of Ecology, Evolution and Systematics 41:59-80. https://doi.org/10.1146/annurev-ecolsys-102209-144650.

Eom, A. H., D. C. Hartnett, and G. W. T. Wilson. 2000. Host plant species effects on arbuscular mycorrhizal fungal communities in tallgrass prairie. Oecologia 122:435-444. https://doi.org/10.1007/s004420050050.

Estrabou, C. 1983. Relevamiento de variantes fisonómicas de la cuenca La Quebrada. Seminarios I y II. Carrera de Ciencias Biológicas, F.C.E.F. y N., Universidad Nacional de Córdoba.

Fernández, R. D., N. Bulacio, A. Álvarez, H. Pajot, and R. Aragón. 2017. Fungal decomposers of leaf litter from an invaded and native mountain forest of NW Argentina. Antonie van Leeuwenhoek 110:1207-1218. https://doi.org/ 10.1007/s10482-017-0893-8.

Fitter, A. H. 2005. Darkness visible: reflections on underground ecology. Journal of Ecology 93:231-243. https://doi.org/ 10.1111/j.0022-0477.2005.00990.x.

Fitzsimons, M. S., R. M. Miller, and J. D Jastrow. 2008. Scale-dependent niche axes of arbuscular mycorrhizal fungi. Oecologia 158:117-127. https://doi.org/10.1007/s00442-008-1117-8.

Faggioli, V. S., M. N. Cabello, G. Grilli, M. Vasar, F. Covacevich, and M. Öpik. 2019.Root colonizing and soil borne communities of arbuscular mycorrhizal fungi differ among soybean fields with contrasting historical land use. Agriculture, Ecosystems and Environment 269:174-182. https:/ /doi.org/10.1016/j.agee.2018.10.002.

Fracchia S., A. Aranda, A. Gopar, V. Silvani, L. Fernández, and A. Godeas. 2009. Mycorrhizal status of plant species in the Chaco Serrano woodland from central Argentina. Mycorrhiza 19:205-214. https://doi.org/10.1007/s00572009-0231-8.

Furey, C., P. A. Tecco, N. Pérez-Harguindeguy, M. A. Giorgis, and M. Grossi. 2014. The importance of native and exotic plant identity and dominance on decomposition patterns in mountain woodlands of central Argentina. Acta Oecologica 54:13-20. https:// doi.org/10.1016/j.actao.2012.12.005.

Gavier-Pizarro, G. I., T. Kuemmerle, L. E. Hoyos, S. I. Stewart, C. D. Huebner, N. S. Keuler, and V. C. Radeloff. 2012. Monitoring the invasion of an exotic tree (Ligustrum lucidum) from 1983 to 2006 with Landsat TM/ETM+ satellite data and Support Vector Machines in Córdoba, Argentina. Remote Sensing of Environment 122:134-145. https: //doi.org/10.1016/j.rse.2011.09.023.

Giorgis, M. A, A. M. Cingolani, D. E. Gurvich, P. A. Tecco, J. Chiapella, F. Chiarini, and M. Cabido. 2017. Changes in floristic composition and physiognomy are decoupled along elevation gradients in central Argentina. Applied Vegetation Science 20:558-571. https:// doi.org/10.1111/avsc.12324.

Greipsson, S., and A. DiTommaso. 2006. Invasive non-native plants alter the occurrence of arbuscular mycorrhizal 
fungi and benefit from this association. Ecological Restoration 24:236-241. https://doi.org/10.3368/er.24.4.236.

Grilli, G., C. Urcelay, and L. Galetto. 2012. Forest fragment size and nutrient availability: complex responses of mycorrhizal fungi in native-exotic hosts. Plant Ecology 213:155-165. https://doi.org/10.1007/s11258-011-9966-3.

Grime, J. P. 1977. Evidence for the existence of three primary strategies in plants and its relevance to ecological and evolutionary theory. The American Naturalist 111:1169-1194. https://doi.org/10.1086/283244.

Guisande-Collazo, A., L. González, and P. Souza-Alonso. 2016. Impact of an invasive nitrogen-fixing tree on arbuscular mycorrhizal fungi and the development of native species. AoB Plants 8. https://doi.org/10.1093/aobpla/plw018.

Helgason, T., J. W. Merryweather, J. Denison, P. Wilson, and J. P. W. Young. 2002. Selectivity and functional diversity in arbuscular mycorrhizas of co-occurring fungi and plants from a temperate desiduous woodland. Journal of Ecology 90:371-384. https://doi.org/10.1046/j.1365-2745.2001.00674.x.

van der Heyde, M., B. Ohsowski, L. K. Abbott, and M. Hart. 2017. Arbuscular mycorrhizal fungus responses to disturbance are context-dependent. Mycorrhiza 27:431-440. https://doi.org/10.1007/s00572-016-0759-3.

Hiiesalu, I., M. Pärtel, J. Davison, P. Gerhold, M. Metsis, M. Moora, M. Opik, M. Vasar, M. Zobel, and S. D. Wilson. 2014. Species richness of arbuscular mycorrhizal fungi: associations with grassland plant richness and biomass. New Phytologist 203:233-244. https://doi.org/10.1111/nph.12765.

Hobbie, S. E. 1992. Effects of plant species on nutrient cycling. Trends in Ecology and Evolution 7:336-339. https: //doi.org/10.1016/0169-5347(92)90126-V.

Hoyos, L. E., G. I. Gavier-Pizarro, T. Kuemmerle, E. H. Bucher, V. C. Radeloff, and P. A. Tecco. 2010. Invasion of glossy privet (Ligustrum lucidum) and native forest loss in the Sierras Chicas of Córdoba, Argentina. Biological Invasions 12: 3261-3275. https://doi.org/10.1007/s10530-010-9720-0.

Jansa, J., A. Erb, H. R. Oberholzer, P. Šmilauer, and S. Egli. 2014. Soil and geography are more important determinants of indigenous arbuscular mycorrhizal communities than management practices in Swiss agricultural soils. Molecular Ecology 23:2118-2135. https:// doi.org/10.1111/mec.12706.

Kiers, E. T., M. Duhamel, Y. Beesetty, J. A Mensah, O. Franken, E Verbruggen, R. C. Fellbaum, A. G. Kowalchuk, M. M. Hart, A. Bago, T. M. Palmer, S. A. West, P. Vandenkoornhuyse, and J. Jansa. 2011. Reciprocal rewards stabilize cooperation in the mycorrhizal symbiosis. Science 333:880-882. https://doi.org/10.1126/science.1208473.

Liao, C., R. Peng, Y. Luo, X. Zhou, X Wu, C. Fang, C., J. Chen, and B. Li. 2008. Altered ecosystem carbon and nitrogen cycles by plant invasion: a metaDanalysis. New Phytologist 177:706-714. https://doi.org/10.1111/j.14698137.2007.02290.x.

Longo, S., E. Nouhra, B. T. Goto, R. L. Berbara, and C. Urcelay. 2014. Effects of fire on arbuscular mycorrhizal fungi in the Mountain Chaco Forest. Forest Ecology and Management 315:86-94. https://doi.org/10.1016/j.foreco.2013.12.027.

Longo, S., N. Cofré, F. Soteras, G. Grilli, M. Lugo, and C. Urcelay. 2016. Taxonomic and Functional Response of Arbuscular Mycorrhizal Fungi to Land Use Change in Central Argentina. Pp. 81-90 en M. C. Pagano (ed.). Recent advances on mycorrhizal fungi. Springer. Belo Horizonte. Minas Gerais. Brazil. https:/ / doi.org/10.1007/978-3-31924355-9_7.

Lovelock, C. E., K. Andersen, and J. B. Morton. 2003. Arbuscular mycorrhizal communities in tropical forests are affected by host tree species and environment. Oecologia 135:268-279. https://doi.org/10.1007/s00442-002-1166-3.

Lutti, R., M. Solis, M Galera, N Muller, and M. Berzal. 1979. Vegetación. Pp. 297-368 en J. Vázquez, R. Miatello and M. Roque (eds.). Geografía Física de la Provincia de Córdoba. Boldt. Buenos Aires. Argentina.

Maherali, H., and J. N. Klironomos. 2007. Influence of phylogeny on fungal community assembly and ecosystem functioning. Science 316:1746-1748. https://doi.org/10.1126/science.1143082.

Moglia, G., and A. M. Giménez. 1998. Rasgos anatómicos característicos del hidrosistema de las principales especies arbóreas de la región chaqueña argentina. Forest Systems 7:53-71.

Moyano, J., M. A. RodríguezロCabal., and M. A. Núñez. 2020. Highly invasive tree species are more dependent on mutualisms. Ecology 101(5):e02997. https://doi.org/10.1002/ecy.2997.

Núñez, M. A., and I. A. Dickie. 2014. Invasive belowground mutualists of woody plants. Biological Invasions 16:645661. https://doi.org/10.1007/s10530-013-0612-y.

Oksanen, J., F. G. Blanchet, M. Friendly, R. Kindt, P. Legendre, D. McGlinn, P. R. Minchin, R. B. O'Hara, G. L. Simpson, P. Solymos, M. H. H. Stevens, E. Szoecs, and H. Wagner. 2019. Package 'vegan': Community Ecology Package. R package version 2.5-6

Pyšek, P., V. Jarošík, P. E Hulme, J. Pergl, M. Hejda, U. Schaffner, and M. Vilà. 2012. A global assessment of invasive plant impacts on resident species, communities and ecosystems: the interaction of impact measures, invading species' traits and environment. Global Change Biology 18:1725-1737. https://doi.org/10.1111/j.1365-2486.2011.02636.x.

R Core Team. 2019. R: A language and environment for statistical computing. R Foundation for Statistical Computing, Vienna, Austria. URL: https://www.R-project.org/.

Redecker D., A. Schüßler, H. Stockinger, S. L. Stürmer, J. B. Morton, and C. Walker. 2013. An evidence-based consensus for the classification of arbuscular mycorrhizal fungi (Glomeromycota). Mycorrhiza 23:515-531. https://doi.org/ 10.1007/s00572-013-0486-y.

Reinhart, K. O., and R. M. Callaway. 2006. Soil biota and invasive plants. New Phytologist 170:445-457. https://doi.org/ 10.1111/j.1469-8137.2006.01715.x.

Richardson, D. M., C. Hui, M. A. Núñez, and A. Pauchard. 2014. Tree invasions: patterns, processes, challenges and opportunities. Biological Invasions 16:473-81. https://doi.org/10.1007/s10530-013-0606-9.

Richardson, D. M., P. Pyšek, M. Rejmánek, M. G. Barbour, F. D. Panetta, and C. J. West. 2000. Naturalization and 
invasion of alien plants: concepts and definitions. Diversity and distributions 6:93-107. https://doi.org/10.1046/ j.1472-4642.2000.00083.x.

Río, M. E., and L. Achával. 1904. Geografía de la Provincia de Córdoba.

Roberts, D. W. 2016. labdsv: Ordination and Multivariate Analysis for Ecology. R package version 1.8-0.

Sala, O. E., F. S. Chapin, J. J. Armesto, E. Berlow, J. Bloomfield, R. Dirzo, E. Huber-Sanwald, L. F. Huenneke, R. B. Jackson, A. Kinzig, R. Leemans, D. M. Lodge, H. A. Mooney, M. Oesterheld, N. LeRoy Poff, M. T. Sykes, B. H. Walker, M. Walker, and D. H. Wall. 2000. Global biodiversity scenarios for the year 2100. Science 287:1770-1774. https://doi.org/10.1126/science.287.5459.1770.

Scheublin, T. R., R. S. van Logtestijn, and M. G. van Der Heijden. 2007. Presence and identity of arbuscular mycorrhizal fungi influence competitive interactions between plant species. Journal of Ecology 95:631-638. https://doi.org/ 10.1111/j.1365-2745.2007.01244.x.

Schüßler, A., and C. Walker. 2010. The Glomeromycota: a species list with new families and new genera. Gloucester, in libraries at The Royal Botanic Garden Edinburgh, The Royal Botanic Garden Kew, Botanische Staatssammlung Munich and Oregon State University.

Sharmah, D., and D. K Jha. 2014. Diversity of arbuscular mycorrhizal fungi in disturbed and undisturbed forests of Karbi Anglong Hills of Assam, India. Agricultural Research 3:229-238. https://doi.org/10.1007/s40003-014-0110-1.

Sikes, B. A., K. Cottenie, and J. N. Klironomos. 2009. Plant and fungal identity determines pathogen protection of plant roots by arbuscular mycorrhizas. Journal of Ecology 97:1274-1280. https://doi.org/10.1111/j.13652745.2009.01557.x.

Smith, S. E., and D. J. Read. 2008. Mycorrhizal symbiosis. 3rd. Academic Press, New York.

Sparks, D. L. 1996. Methods of soil analysis. Part 3. Chemical methods. ASA, SSSA, CSSA, Madison, WI. https:// doi.org/10.2136/sssabookser5.3.

Tecco, P. A., C. Urcelay, S. Díaz, M. Cabido, and N. PérezDHarguindeguy. 2013. Contrasting functional trait syndromes underlay woody alien success in the same ecosystem. Austral Ecology 38:443-451. https://doi.org/10.1111/j.14429993.2012.02428.x.

Treseder, K. K. 2004. A metalanalysis of mycorrhizal responses to nitrogen, phosphorus, and atmospheric CO2 in field studies. New Phytologist 164:347-355. https://doi.org/10.1111/j.1469-8137.2004.01159.x.

Urcelay, C., S. Díaz, D. E. Gurvich, F. S. Chapin III, E. Cuevas, and L. S. Domínguez. 2009. Mycorrhizal community resilience in response to experimental plant functional type removals in a woody ecosystem. Journal of Ecology 97 : 1291-1301. https://doi.org/10.1111/j.1365-2745.2009.01582.x.

Urcelay, C., S. Longo, J. Geml, and P. A. Tecco. 2019. Can arbuscular mycorrhizal fungi from non-invaded montane ecosystems facilitate the growth of alien trees? Mycorrhiza 29:39-49. https://doi.org/10.1007/s00572-018-0874-4.

Yang, H., Z. Yanyan, Y. Yongge, T. Jianjun, and C. Xin. 2012. Selectivity by host plants affects the distribution of arbuscular mycorrhizal fungi: Evidence from ITS rDNA sequence metadata. Evolutionary Biology 12:50. https: //doi.org/10.1186/1471-2148-12-50.

Yang, H., J. Xu, Y. Guo, R. T. Koide, Y. Dai, M. Xu, L. Biand, X. Bia, and Q. Zhang. 2016. Predicting plant response to arbuscular mycorrhizas: the role of host functional traits. Fungal Ecology 20:79-83. https://doi.org/10.1016/ j.funeco.2015.12.001.

Zeballos, S. R., M. A. Giorgis, A. M. Cingolani, M. Cabido, J. I. Whitworth Hulse, and D. E. Gurvich. 2014. Do alien and native tree species from Central Argentina differ in their water transport strategy? Austral Ecology 39:984-991. https://doi.org/10.1111/aec.12171.

Zhang, Q., R. Yang, J. Tang, H. Yang, S. Hu, and X. Chen. 2010. Positive feedback between mycorrhizal fungi and plants influences plant invasion success and resistance to invasion. PLoS ONE 5(8). https://doi.org/10.1371/ journal.pone.0012380. 